\title{
Psicosis postictal con delusiones de contenido religioso: reporte de caso.
}

Postictal psychosis with religious delusions: a case report.

\author{
Elliot Barreto-Acevedo ${ }^{1,2, \text { a }}$, Leila Barreto-Barra ${ }^{1,}$, Mirla Villafuerte-Espinoza ${ }^{1,2, \text { a, }}$ \\ Alberto Díaz- Vásquez ${ }^{1, a}$, Alicia Becerra-Zegarra ${ }^{3, \text { b }}$, Hernández-Vizarreta José ${ }^{1, a}$, Victoria Llaja-Rojas ${ }^{1,4, c}$.
}

\section{RESUMEN}

Desde la antigüedad se han descrito manifestaciones de religiosidad peri-ictal en epilepsia. Es frecuente la psicopatología asociada a epilepsia; entre otras manifestaciones, la psicosis asociada a epilepsia ha sido reportada aunque con menor frecuencia que la depresión y la ansiedad; en especial la psicosis postictal. El uso del monitoreo prolongado con Video- EEG en los últimos años ha permitido estudiar mejor estas manifestaciones. Presentamos un caso de epilepsia del lóbulo temporal farmacorresistente, con un episodio de psicosis postictal caracterizado por delusiones de contenido religioso. Se realizó un estudio detallado que incluyó resonancia de encéfalo y Video-EEG prolongado; revisamos la literatura y los mecanismos fisiopatogénicos propuestos para este fenómeno postictal.

PALABRAS CLAVE: Epilepsia del lóbulo temporal, religiosidad, psicosis postictal, Video-EEG.

\section{SUMMARY}

Peri-ictal religious manifestations in epilepsy have been described since ancestral times. Psychopathology associated with epilepsy is frequent; among other manifestations, the psychosis associated with epilepsy has been reported, although less frequently than depression and anxiety; especially postictal psychosis. In recent years the use of prolonged monitoring with Video-EEG has allowed us to better study these manifestations. We present a case of drug-resistant temporal lobe epilepsy, with an episode of postictal psychosis characterized by religious delusions. A detailed study was performed that included brain imaging and prolonged Video-EEG. We review the literature and physiopathogenic mechanisms proposed for this postictal phenomenon.

KEYWORDS: Temporal lobe epilepsy, religiosity, postictal psychosis, Video-EEG.

1 Unidad de Epilepsia, Departamento de Neurología, Hospital Nacional Edgardo Rebagliati Martins, EsSalud. Lima, Perú.

2 Facultad de Medicina, Universidad Nacional Mayor de San Marcos. Lima, Perú.

3 Servicio de Neurocirugía Funcional, Hospital Nacional Edgardo Rebagliati Martins, EsSalud. Lima, Perú.

4 Facultad de Psicología, Universidad Nacional Mayor de San Marcos. Lima, Perú.

a Médico-Neurólogo; ${ }^{\mathrm{b}}$ Médico-Neurocirujano de Epilepsia; ${ }^{\mathrm{c}}$ Neuropsicóloga. 


\section{INTRODUCCIÓN}

Los síntomas psicóticos en pacientes con epilepsia han sido descritos desde mediados del siglo XIX, reportándose casos de "locura" asociada a epilepsia. Jackson consideraba que la epilepsia era causa de "locura" (Jackson, 1875) y que el 6\% de los pacientes en instituciones mentales debían su "locura" a la epilepsia (1). Asi mismo desde la antigüedad se ha tratado de entender la asociación entre religiosidad y epilepsia. A pesar de los intentos de Hipócrates de disociar la epilepsia y la religión; en el siglo XVI y XVII, durante la Cristiandad, se le dio una connotación de posesión demoniaca a la epilepsia. En el siglo XIX, sin embargo, la religiosidad en la epilepsia atrajo el interés científico de neurólogos y psiquiatras en instituciones mentales en Europa $(2,3,4)$. Desde el siglo pasado, con el advenimiento del electroencefalograma (EEG) y posteriormente del Video-EEG, se han caracterizado mejor las manifestaciones religiosas en epilepsia. Estas manifestaciones han sido reportadas con escasa frecuencia en la literatura $(0,4$ a $4 \%)$ y las siguientes presentaciones semiológicas han sido descritas: Auras de éxtasis o alucinaciones auditivas $\mathrm{y}$ visuales de contenido religioso, delusiones y experiencias religiosas en el postictal, hiperreligiosidad interictal, trastorno de personalidad caracterizado por hiperreligiosidad, hipergrafía, alteración de la sexualidad, viscosidad en el pensamiento y disforia, denominado síndrome de Gastaut-Geschwind; rezo o plegaria ictal y automatismos ictales gestuales de rezo como manos en posición de plegaria, el signo de la cruz o postración religiosa (4-11).

Lapsicopatología es más prevalente en los pacientes con epilepsia que en la población en general. Se ha descrito una mayor prevalencia de depresión, ansiedad, trastorno de personalidad y psicosis en pacientes con epilepsia. La asociación de manifestaciones psicóticas a la epilepsia, han sido estudiadas tanto como fenómenos postictales e interictales. Se han reportado manifestaciones de religiosidad como parte de psicosis postictal $(3,12-15)$. Presentamos un caso de epilepsia del lóbulo temporal farmacorresistente, quien presentó durante el monitoreo prolongado con VideoEEG, un episodio de psicosis postictal con delusiones de contenido exclusivamente religioso. Revisamos la literatura al respecto con los objetivos de conocer la semiología de esta manifestación postictal, los factores de riesgo para su ocurrencia y describir las teorías sobre su fisiopatogenia.

\section{Descripción del caso}

Varón de 43 años, escribe con la mano derecha pero utiliza con destreza la mano izquierda para realizar otras actividades, desocupado, católico practicante, antecedente de traumatismo encéfalocraneano (TEC) severo a los 3 años de edad y padece de ansiedad desde la juventud. Tiene epilepsia focal farmacorresistente, con edad de inicio a los 11 años. Presentaba crisis focales con compromiso de conciencia: aura de déjà vu y miedo, seguidos de parada comportamental con automatismos manuales derechos y automatismos orodeglutorios. Las crisis eran diurnas en vigilia y la frecuencia era de 2 a $3 /$ semana. Ha recibido Carbamazepina y Fenitoína en monoterapia sin éxito. Esquema actual: Levetiracetam $3 \mathrm{~g}$ /día y Lamotrigina $100 \mathrm{mg} /$ día. El paciente ingresó a la Unidad de Epilepsia para evaluación prequirúrgica. El examen neurológico fue normal, la resonancia magnética (RM) de encéfalo evidenció: Esclerosis Mesial Temporal (EMT) Derecha (Figura 1). La evaluación neuropsicológica evidenció: coeficiente intelectual normal y leve compromiso mnésico no verbal; no se pudo establecer la dominancia hemisférica para el lenguaje, por lo que

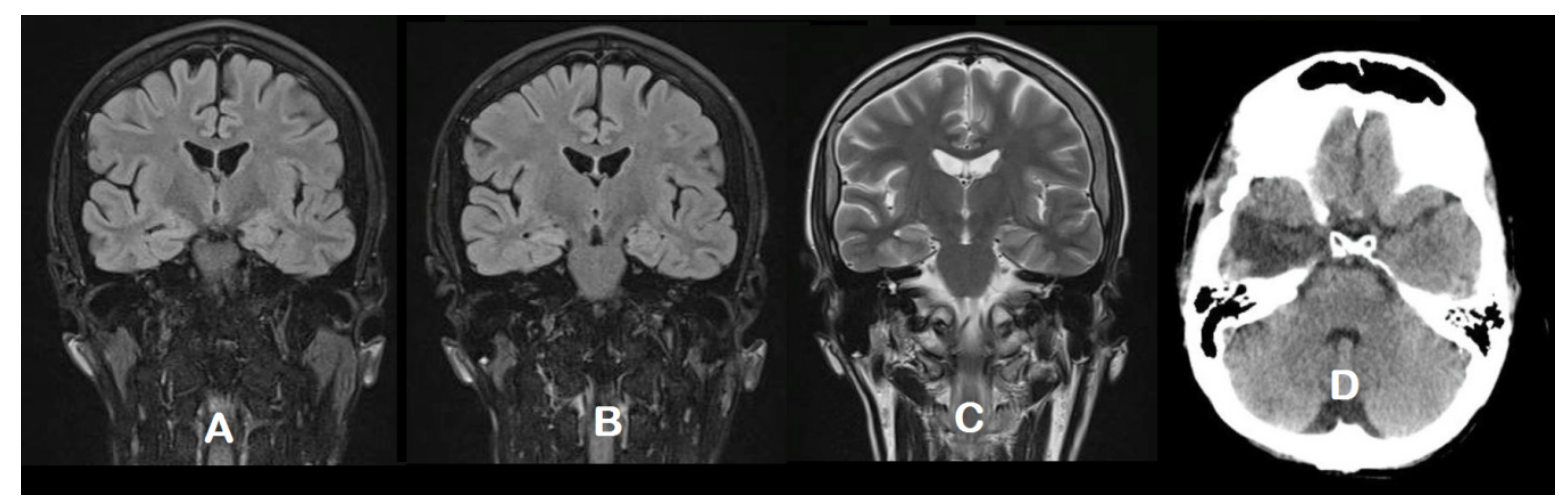

Figura 1.Neuroimagenes. RM de encéfalo: cortes coronales en FLAIR (A y B) y en T2 (C), evidencia EMT derecha. D. Tomografía cerebral postquirúrgica demuestra lobectomía anteromesial derecha. 
se realizó test de Wada con propofol y se determinó dominancia hemisférica izquierda para el lenguaje. Se realizó un Video-EEG de 104 horas que evidenció: frecuente actividad epileptiforme interictal temporal anterior derecha (figura 2), con menor frecuencia actividad bitemporal síncrona a predominio derecho y se registraron 3 crisis diurnas durante vigilia con semiología similar a la descrita en la anamnesis: aura, seguida de parada comportamental, automatismos orales, seguidos automatismos manuales derechos y versión cefálica no forzada hacia la derecha. El EEG ictal evidenció inicio temporal anterior derecho (figura 2). El postictal de las 2 primeras crisis consistió en confusión transitoria con frote nasal con la mano derecha. Pero en la tercera crisis el paciente, luego de una confusión breve y normalización de la conciencia por 3 horas desarrolló psicosis postictal por las siguientes 24 horas. Durante este periodo, el paciente gritaba que: "Dios se le estaba manifestando y que lo había curado, que se sentía con una fuerza nunca antes vista, que se sentía vivo que ya no tenía epilepsia". Así mismo refería "que se había convertido en un discípulo de Jesús y que la cura de su epilepsia estaba escrita en el evangelio de Lucas capítulo 14". Repetía una y otra vez el mismo discurso, juraba que todo lo que decía era cierto, a la vez abrazaba a su familiar intermitentemente, se mostraba hiperactivo, con conducta hipomaniaca, verborreico y se tornaba agresivo cuando intentaban sujetarlo. Se quería levantar de la cama e irse a su casa, pues manifestaba que ya no requería más estudios ni tratamientos (figura 3). El EEG durante la psicosis postictal solo evidenció leve lentificación fronto-temporal derecha. Esta lentificación fue notoriamente más discreta que

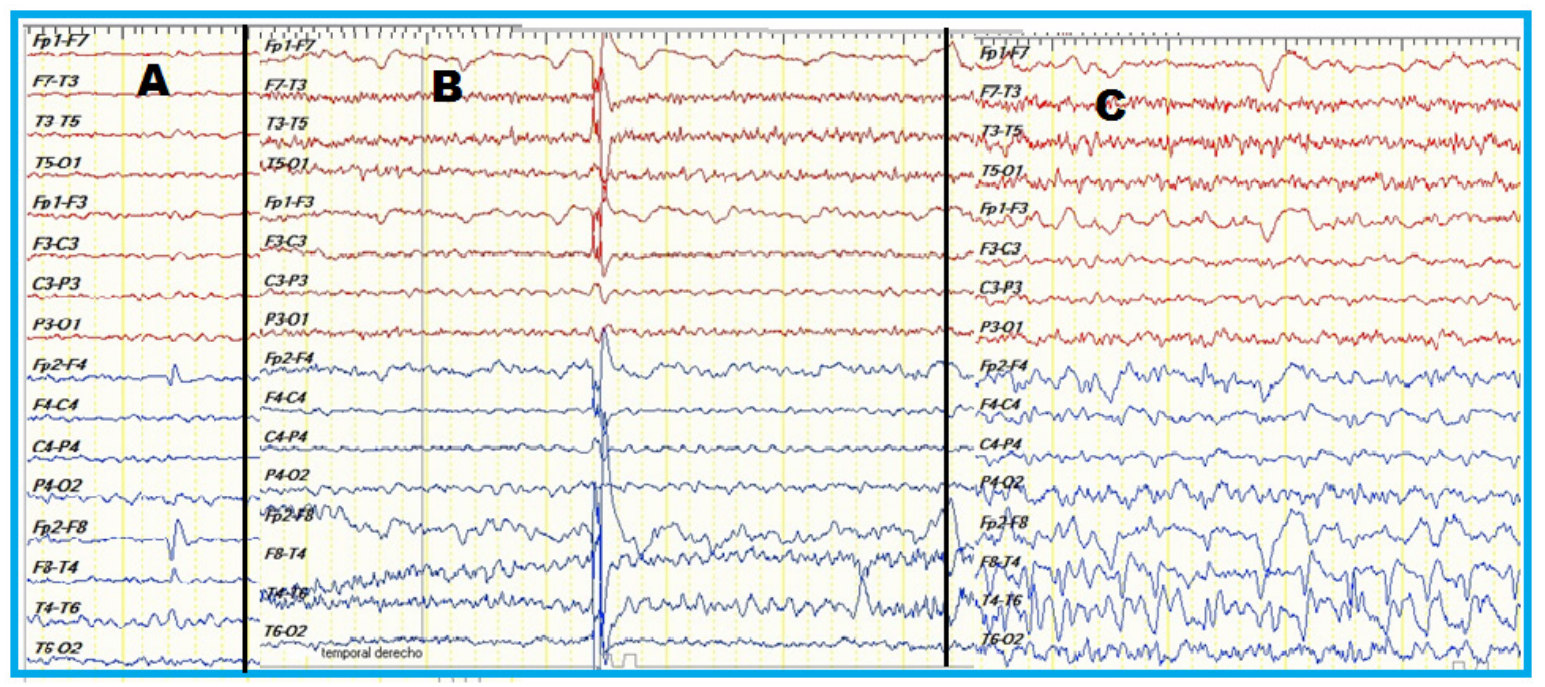

Figura 2. EEG. A: Interictal (punta electronegativa con inversión de fase en F8). B y C: Ictal en el inicio (actividad theta rítmica temporal anterior -media derecha) y en el curso de la crisis.

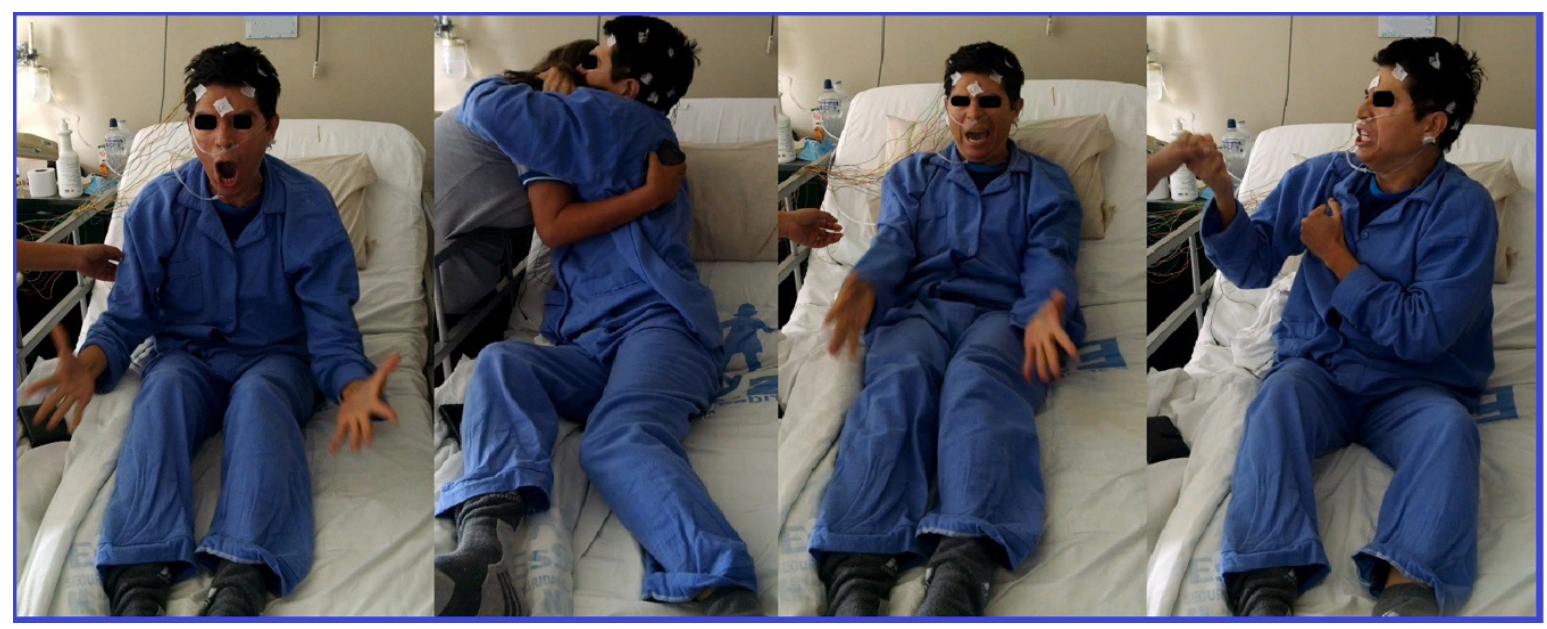

Figura 3. Conducta hiperactiva e hipomaniaca durante la psicosis postictal. 


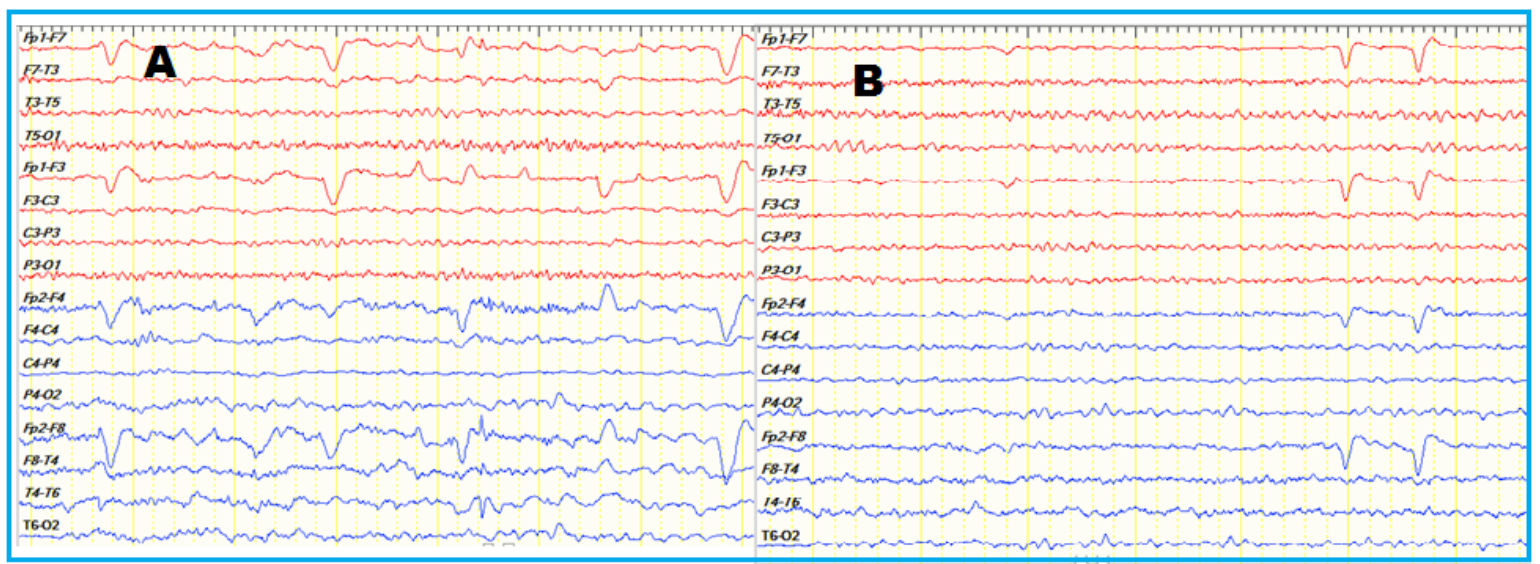

Figura 4. EEG. A: Postictal (marcada lentificación fronto-temporal derecha). B: Durante la psicosis postictal (sutil lentificación temporal derecha).

la observada en el postictal inmediato. (figura 4). El cuadro remitió en 24 horas con administración de 5 $\mathrm{mg}$ de Haloperidol endovenoso y Risperidona $2 \mathrm{mg}$ VO tid, este último fármaco lo recibió por 1 semana. El paciente fue intervenido de una lobectomía anteromesial derecha, la patología demostró esclerosis mesial, no ha desarrollado psicosis interictal, pero su ansiedad ha empeorado y se encuentra en Engel Ia (completamente libre de crisis) después de 1 año.

\section{DISCUSIÓN}

La literatura sobre psicosis postictal con manifestaciones exclusivas de religiosidad es escasa. Las series sobre psicosis postictal suelen incluir reportes de pacientes con manifestaciones no exclusivamente religiosas, este fenómeno fue formalmente descrito como se le conoce en la actualidad por Logsdail en 1988 (13). La psicosis postictal es un fenómeno con baja prevalencia (6 a $10 \%$ de los pacientes de las unidades de monitoreo de epilepsia), reportada principalmente en epilepsias focales temporales y con menor frecuencia en epilepsias extratemporales (13, 16,17). Algunos de los criterios de Logsdail y Toone, señalan que este fenómeno, tal como ocurrió en nuestro paciente, se presenta dentro de una semana de ocurrida una crisis o salva de crisis, con un intervalo lúcido (al menos de 2 a 6 horas y hasta 24 a 48 horas) entre el término de la crisis y el inicio de la psicosis; y dura al menos 15 horas, pudiendo extenderse por días y en ocasiones hasta 2 meses. La duración en nuestro caso fue de aproximadamente de 24 horas, con un intervalo lucido de 3 horas. Las manifestaciones son: alucinaciones usualmente visuales, menos frecuentemente auditivas, sin compromiso del nivel de conciencia; con delirio, delusiones de grandeza y/o de contenido religioso, conducta desorganizada, agresiva o disfórica y cambios afectivos $(1,13,17)$. A diferencia de la psicosis interictal, la psicosis postictal exhibe pocas manifestaciones esquizofreniformes (delusiones de daño o referencia y de persecución) $(1,16)$. Las delusiones y alucinaciones se suelen acompañar de hiperactividad, súbitas conductas agresivas no provocadas, respuestas emocionales excesivamente desproporcianadas a estímulos externos y lenguaje o conducta hipomaniaca. Suele haber un periodo de latencia de alrededor de 15 a 20 años entre el inicio de la epilepsia y la ocurrencia de la psicosis postictal $(1,13,16,17)$. La mayoría de estas características se pueden identificar en nuestro caso.

Devinsky, en su revisión sobre religión y epilepsia, recopila algunos casos descritos en la literatura del siglo IX y siglo XX; en los que las manifestaciones clínicas de religiosidad después de la ocurrencia de crisis epilépticas sugerirían psicosis postictal; por ejemplo, describe el caso de una conversión religiosa después de una crisis generalizada en la cual "el paciente mencionaba estar en el cielo" y ésta experiencia de despersonalización duró 3 días, luego de lo cual el paciente mencionaba que "recién su alma había retornado a su cuerpo, que por primera vez había sentido la paz y que era un nuevo hombre". Así mismo, Devinsky relata otros dos casos con manifestaciones religiosas postictales: uno, el de un paciente quien decía que "Dios le había dado la misión de traer la ley a la tierra y que El y la Virgen María lo comandaban"; y otro, el de un joven de 14 años que decía "ver a Dios, a los Ángeles y que oía una música celestial". El mismo autor describe dos pacientes suyos con psicosis postictal con manifestaciones religiosas. Un caso fue el de un varón de 45 años con 
crisis focales con auras de de'ja 'vu, que a los 27 años presentó un episodio de psicosis postictal con la visión de Jesucristo, y luego oyó una voz que le ordenaba matar a su esposa y matarse así mismo. Apuñaló a su esposa, matándola, luego se apuñaló e incendió su casa. El paciente no murió pero fue recluido en una prisión para pacientes psiquiátricos. Luego del evento no desarrolló psicosis interictal pero se mantuvo con tendencia a la hiperreligiosidad. Los estudios prequirúrgicos determinaron que tenía EMT derecha y zona epileptogena (ZE) temporomesial derecha, fue operado, quedó libre de crisis y sin recurrencia de delusiones o conducta de hiperreligiosidad. El otro caso que describe es el de una mujer de 38 años con epilepsia del lóbulo temporal izquierda por displasia cortical focal (DCF), con descargas interictales bitemporales. La paciente tuvo varios episodios de psicosis postictal, usualmente después de crisis focales en salva; con delusiones que giraban en torno a "la presencia del anticristo; sentía que estaba perdiendo su salvación" y solía preguntar si el número 66 que figuraba como parte de su número de seguro social significaba algo. Fue intervenida de una lobectomía temporal izquierda, con reducción significativa de las crisis y resolución completa de las manifestaciones religiosas postictales (18). Ya en 1963, Beard describió 6 casos de conversión religiosa en pacientes con epilepsia y delusiones de contenido religioso postictales, en todos ellos el común denominador era la ocurrencia de frecuentes crisis con compromiso de conciencia y en la mayoría de ellos la actividad epileptiforme fue bilateral (19).

En la serie de Savard et al, de 9 casos con epilepsia focal que presentaron psicosis postictal, se reportaron 2 pacientes mujeres de 39 y 19 años con epilepsia del lóbulo temporal (ELT) y manifestaciones postictales de delusiones exclusivamente religiosas: La primera paciente refería que Dios se había reencarnado en ella, que tenía poderes magnéticos, mediante los cuales emitía ondas cerebrales que le permitían curar mágicamente a otras personas con epilepsia. El episodio de psicosis remitió después de pocos días tras la administración de haloperidol. La segunda paciente, luego de una salva de crisis focales, viró hacia un comportamiento extraño, solicitó insistentemente una prueba de embarazo, pues alegaba estar embarazada de Dios. Decía que su epilepsia era una prueba de que su cuerpo estaba siendo manejado por el demonio. Recibió Haloperidol $20 \mathrm{mg} /$ día y el episodio de psicosis remitió en 3 semanas (20).

En la psicosis postictal, el EEG suele evidenciar enlentecimiento difuso o regional, como fue el caso de nuestro paciente; pero también puede evidenciar un incremento de la actividad interictal habitual. Con menor frecuencia se ha descrito el fenómeno de normalización forzada, que consiste en la supresión de la actividad epileptiforme interictal durante el periodo de psicosis $(1,17)$. En base a escasos estudios con electrodos profundos, en los que se ha encontrado actividad eléctrica ictal residual temporal, algunos autores han planteado que la psicosis postictal podría tratarse, en ciertos pacientes, de un estado epiléptico focal no convulsivo $(21,22)$. Se han descrito factores de riesgo de psicosis postictal como: crisis focales con compromiso de conciencia en salva o crisis generalizadas previo al evento, antecedente de TEC, encefalitis $u$ otras lesiones que impliquen compromiso encefálico bilateral, historia familiar de enfermedad psiquiátrica, actividad eléctrica bilateral y epilepsia temporal bilateral $(1,13,16,17)$ Tres de estos factores fueron evidenciados en nuestro paciente. Los estudios no han encontrado en forma uniforme una etiología de la epilepsia asociada a psicosis postictal, se han descrito casos asociados esclerosis hipocampal como el nuestro, pero también casos por DCF, tumores y otras etiologías $(17,19)$. Aunque no ha sido estudiada en forma sistemática y exclusiva esta manifestación como signo lateralizador o focalizador, hay varios estudios que reportan que esta manifestación es más frecuente, aunque no es exclusiva del lóbulo temporal y además no tendría un valor lateralizador $(1,16,17,20)$. Kanemoto, en 30 casos de psicosis postictal en pacientes con epilepsia focal, encontró que el foco epileptógeno era temporal en 24 casos, frontal en 4 casos, parietal en 1 caso y occipital en 1 caso; en tanto que la lateralidad fue izquierda en 9 casos, derecha en 11 casos y no se pudo lateralizar en 10 casos (16).

El $50 \%$ de los pacientes con psicosis postictal responden bien a los antipsicóticos atípicos dentro del periodo de 1 semana, a diferencia de una pobre respuesta en la psicosis interictal. La asociación a benzodiacepinas mejora su respuesta. El tratamiento precoz de la psicosis postictal favorece una rápida respuesta, como quedó demostrado en nuestro caso $(1,16,17,20)$. La cirugía resectiva de la ZE permite también la resolución de la psicosis postictal, pero en el caso de lobectomías temporales, el antecedente de este fenómeno es un factor de riesgo para el desarrollo postquirúrgico de otras patologías afectivas (17). Nuestro paciente, quien previo a la cirugía ya padecía de ansiedad; presentó un empeoramiento de su trastorno afectivo posterior a la cirugía. De un 12 a $50 \%$ podrían tener psicosis postictal recurrente y estos 
pacientes podrían progresar a psicosis interictal $(17$, 22). Nuestro paciente respondió bien al tratamiento con antipsicóticos y no presentó nuevos eventos de psicosis posterior a la cirugía, recibiendo tratamiento con antipsicóticos solo por 1 semana.

Algunos estudios han soportado la hipótesis general de que las manifestaciones de religiosidad en epilepsia, serian fenómenos cognitivo-emocionales generados por activación de redes neuronales límbicas $(4,10,14)$. Sin embargo, es poco probable que se trate de un fenómeno puramente neurobiológico y es más probable, que estas manifestaciones de religiosidad sean además, influenciadas por factores sociales, como lo sugieren la mayoría de reportes y series de casos de manifestaciones religiosas peri-ictales, que han sido descritas en comunidades con profundo arraigo religioso $(9,10,11)$.

La fisiopatogenia de esta manifestación probablemente también tenga que ver con activación crónica de redes neuronales límbicas como lo plantea Wieser; avalado por la fenomenología de contenido emocional (delusiones de grandeza o de religiosidad) que se reporta en la psicosis postictal a diferencia de la psicosis interictal y por su mayor ocurrencia en ELT (21).

Kanemoto, siguiendo la teoría de Wolf, en cambio plantea que en estos pacientes el generador de las descargas epilépticas se mantiene aún activo en el "postictal" pero que la vía usual de propagación ha sido bloqueada, resultando en un nuevo patrón de propagación que conllevaría a los síntomas psicóticos (16). El reporte de Kuba, de 2 pacientes con ELT EMT y psicosis postictal, estudiados con electrodos profundos en quienes, durante la psicosis se encontró un patrón epileptiforme continuo diferente de la actividad interictal e ictal habitual (actividad lenta rítmica hipocampal derecha y ondas trifásicas en el cíngulo anterior izquierdo), reforzaría la hipótesis de Kanemoto - Wolf (22). Por otro lado, Devinski plantea, en base a ciertas características de la psicosis postictal: frecuente patología estructural bilateral y redes epileptógenas bilaterales, antecedentes familiares de enfermedad psiquiátrica, periodo lucido previo a la psicosis y hallazgos de hipermetabolismo cerebral durante la psicosis; la posibilidad de un rebote de la actividad eléctrica excesiva después de la depresión postictal. Este rebote de hiperactividad cortical sostenida en pacientes con disfunción cerebral bilateral y predisposición genética a enfermedad psiquiátrica, podría favorecer la ocurrencia de la psicosis postictal al comprometerse la regulación de redes de control cognitivo-emocionales fronto - temporales bilaterales (17). Resulta interesante 2 observaciones en la semiología de la psicosis postictal: el intervalo lúcido que iría en contra de que la psicosis postictal sea solo una extensión de la confusión postictal inmediata o una continuidad de la actividad epiléptica ictal y la latencia habitual de 15 a 20 años desde el inicio de la epilepsia para el desarrollo de la psicosis postictal. Esto último, sugeriría la necesidad de una activación repetida, sostenida y crónica de redes epileptógenas para desencadenar este fenómeno. Estas observaciones refuerzan la hipótesis de Wieser (21). Finalmente no descartamos la influencia sociocultural para esta manifestación, como ocurre en otras manifestaciones de hiperreligiosidad peri-ictal, pero se requieren más estudios al respecto (11).

En conclusión, la psicosis postictal con contenido de hiperreligiosidad es una manifestación poco frecuente. Es mandatorio el periodo "lúcido" posterior a la crisis y los factores de riesgo para su presentación son: crisis con compromiso de conciencia en alta frecuencia o en salva, lesiones y/o actividad eléctrica cerebral bilateral (en especial bitemporal) y antecedentes familiares de enfermedad psiquiátrica. Sus mecanismos fisiopatogénicos no están del todo esclarecidos; factores psicosociales y neurobiológicos pueden estar involucrados. Se requerirá de un mayor número de reportes y el estudio de las redes neuronales relacionadas con los contenidos cognitivos - emocionales de la religiosidad de estos casos, no solo mediante estudios neurofisiológicos sin también mediante neuro-imágenes funcionales.

Los autores no han recibido financiamiento de ninguna institución. El trabajo ha sido autofinanciado. Los autores no tienen conflicto de interés.

\section{Correspondencia}

Dr. Elliot Barreto Acevedo

Hospital Nacional Edgardo Rebagliati Martins

Av. Rebagliati 490 Jesús María

Teléfono: 511-265-4901 anexo: 3081. Celular: 51989963057.

Correo electrónico: elliotba@hotmail.com

\section{REFERENCIAS BIBLIOGRÁFICAS}

1. Nadkarni S, Arnedo V, Devinsky O. Psychosis in epilepsy patients. Epilepsia, 2007; 48(Suppl. 9):17-9. DOI: $10.1111 /$ j.1528-1167.2007.01394.x 
2. McCrae N, Whitley R. Exaltation in temporal lobe epilepsy: Neuropsychiatric. Symptom or Portal to the Divine? J Med Humanit. 2014; 35:241-55. DOI: 10.1007/s10912-014-9294-4

3. Muhammed L. A retrospective diagnosis of epilepsy in three historical figures: St Paul, Joan of Arc and Socrates. J Med Biogr. 2013; 21: 208-11. DOI: $10.1177 / 0967772013479757$

4. Devinsky O, Lai G. Spirituality and Religion in Epilepsy. Epilepsy Behav. 2008; 12: 636-43.

5. Dong L, Zhou X. An uncommon automatism with religious connotation- postration in a case of right temporal lobe epilepsy. Seizure. 2016; 35: 33-5. DOI: 10.1016/j.seizure.2015.12.012

6. Özkara C, Sarý H, Hanoglu L, Yeni N. Aydogdu I, Özyurt E. Ictal kissing and religious speech in a patient with right temporal lobe epilepsy. Epileptic Disord. 2004; 6: 241-6.

7. Hansena Band, Brodtkorb E. Partial epilepsy with "ecstatic" seizures. Epilepsy Behav.2003; 4: 66773.

8. Carrazana E, De Toledo J, Tatum W, Rivas-Vasquez R, Rey G, Wheeler S. Epilepsy and Religious Experiences: Voodoo Possession. Epilepsia. 1999; 40(2):239-241.

9. Besocke A, Baccanelli M, Cristiano E, García MC, Silva W, Valiensi S. Manifestaciones religiosas como semiología ictal en la epilepsia del lóbulo temporal. Rev Neurol. 2012; 54 (1): 61-3.

10. Lin K, Marx C, Caboclo L, Centeno R, Sakamoto A, Yacubian E. Sign of the cross (signum crucis): observation of an uncommon ictal manifestation of mesial temporal lobe epilepsy. Epilepsy Behav. 2009; 14: 400-3.

11. Barreto-Acevedo E, Barreto-Barra L, VillafuerteEspinoza M, Diaz-Vasquez A, Becerra-Zegarra A, Hernandez-Vizarreta J. El infrecuente signo de la cruz en epilepsia del lóbulo temporal: reporte de caso documentado con video-EEG. Rev Neuropsiquiatr. 2018; 81(4):165-170.

12. Patel R, Elmmadawi A, Mansuri Z, Kaur M Shah $\mathrm{K}$, Nasr S. Psychiatric comorbidities and otucomes in epilepsy patients: An insight from a Nationwide Inpatient Analysis in the United Stated. Cureus. 2017; 9(9):e1686.
13. Logsdail S, Toone B. Post-ictal psychosis: a clinical and phenomenological description. Br J Psychiatry. 1988;152:246-52.

14. Dewhurst K, Beard AW. Sudden religious conversions in temporal lobe epilepsy. Epilepsy Behav. 2003; 4:78-87.

15. Trimble M, Freeman A. An investigation of religiosity and the Gastaut-Geschwind syndrome in patients with temporal lobe epilepsy. Epilepsy Behav. 2006; 9: 407-14.

16. Kanemoto K, Kawasaki J, Kawai I. Postictal Psychosis: A comparison with acute interictal and chronic psychoses. Epilepsia. 1996; 37(6):551-6.

17. Devinsky O. Postictal psychosis: common dangerous and tratable. Epilepsy Currents. 2008; 8(2): 31-4.

18. Devinsky O, Lai G. Spirituality and religion in epilepsy. Epilepsy \& Behavior. 2008; 12: 636-43.

19. Beard AW. The schizophrenia-like psychoses of epilepsy, physical aspects. Brit J Psychiat. 1963;109:113-29.

20. Savard G, Andermann F, Olivier A, Remillard G. Postictal psychosis after partial complex seizures: A multiple case study. Epilepsia. 1991; 32(2):225-231.

21. Wieser HG, Hailemariam S. Regard M, Landis T. Unilateral limbic epileptic status activity: stereo-EEG. behavioural, and cognitive data. Epilepsia. 1985;26:19-29.

22. Kuba R, Brázdil M, Rektor I. Postictal psychosis and its electrophysiological correlates in invasive EEG: A case report study and literature review. Epilepsy \& Behavior. 2012; 23:426-430.

23. Clearya,R, Thompsona P, Thoma M, Foong J. Postictal psychosis in temporal lobe epilepsy: Riskfactors and postsurgical outcome? Epilepsy Researc. 2013; 106: 264-72.

Recibido: 25/02/2019

Aceptado: 31/03/2019 